\title{
Supporting outreach programmes for haemophilia diagnostics
}

Angus McCraw

The Katharine Dormandy haemophilia centre has played an enormous role over many years in providing outreach programmes to centres in developing countries. Doing so offers the potential to improve patient care by enhancing understanding of the need for accurate diagnosis and management.

Keywords: laboratory, outreach, Katharine Dormandy

The Katharine Dormandy Haemophilia Centre outreach programmes to train laboratory technicians in haemophilia came about through having a first-class haemostasis and thrombosis laboratory, being the first laboratory in the Royal Free to be accredited, having well-trained staff who were not afraid to teach and the support of the centre directors.

The reputation of the laboratory has come through World Federation of Hemophilia twinning, Train the Trainer workshop attendees, and the MRCPath courses, which attracted attendees from all over the world. When these students returned to their countries they wanted to do something similar locally, which was how the workshop in Hong Kong started and led to a programme of workshops in the Philippines.

None of this would have been possible without grant funding from the Katharine Dormandy Trust. In addition, we were able to generate income from the haemophilia centre courses, along with support from pharmaceutical and diagnostic companies in the UK and locally in the countries we worked in.

The Hong Kong course for example, has been running since 1994 along with Professor L C Chan and Dr Clarence Lam at the Queen Mary Hospital, University of Hong Kong. It typically attracts an audience of 100 for lectures and 30 for the workshops. The past two workshops have focused on automation and three diagnostic companies were invited to participate. All three ran the same tests such as preparing reference ranges and factor assays and results were compared.

The Philippine courses started in 1997 along with Dr Mary Chua when there were only 100 patients with haemophilia registered in the country. Ten years and ten workshops later, there were around 1000 patients. These workshops became very popular and often word would get out and we would never know how many were going to turn up. Along with the participants, patients would also arrive who would journey for days to get there in order to be investigated. Workshops have also been conducted in

Angus McCraw, Katharine Dormandy Haemophilia Centre \& Thrombosis Unit, Royal Free Hospital, London, UK

Email: ahmccrawabtinternet.com

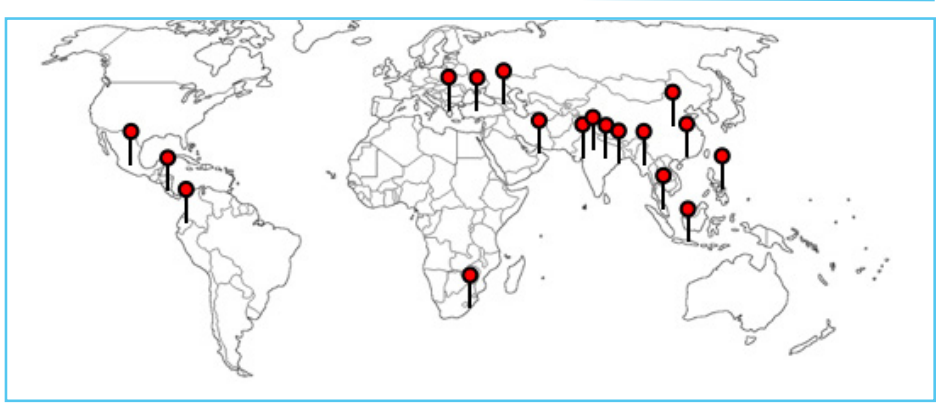

Overseas workshops and Train the Trainers seminars have now taken place across the world

many other countries such as India, Myanmar, Malaysia and Peru. The Train the Trainers workshops run on behalf of WFH also became another door opener, because these doctors then went back to their own countries and conducted workshops. We have run these workshops in Costa Rica, Tbilisi, Mexico, Venezuela and Peru. In 2007 I was approached by the Novo Nordisk Haemophilia Foundation to train three clinicians from China in the diagnosis of inhibitors. I, along with Anne Riddell from the Centre then visited their laboratories to see how they were applying the skills learned during the training.

These outreach programmes improve patient care in developing countries by enhancing understanding of the need for accurate diagnosis in order to support clinicians to give appropriate treatment and thus improve their management. They are a major part of the history and culture of the Katharine Dormandy Haemophilia Centre.

\section{Disclosures}

The scientific meeting on which this issue of The Journal of Haemophilia Practice is based was sponsored by Baxter, Bayer, CSL Behring, Grifols, Novo Nordisk, Sobi, Pfizer, BPL and Werfen. Editorial support for the article was provided by the publisher.

\section{Angus McCraw}

Angus McCraw retired in December 2011 as Principal Biomedical Scientist and Service Manager at the Katharine Dormandy Haemophilia Centre. Dr McCraw initially trained as a laboratory scientist at the Edinburgh Royal Infirmary, after which he ran the coagulation laboratory at Hammersmith Hospital, London, where he ran the coagulation module for the Diploma in Clinical Pathology. He joined the Royal Free in 1981, where in addition to extensive teaching and training responsibilities in diagnosis of inherited bleeding and thrombotic disorders, he began running training workshops in the developing world. Dr McCraw was one of the first recipients of the Inga Marie Nilsson Award in 2004, obtained a doctorate from Middlesex University in 2006 and was appointed Associate Professor of Pathology, University of Hong Kong in January 2009. He is an active member of the WFH and continues to conduct coagulation workshops in developing countries and lectures on laboratory science in many parts of the world. 